\title{
Viable mice produced from three-factor induced pluripo- tent stem (iPS) cells through tetraploid complementation
}

Cell Research (2011) 21:546-549. doi:10.1038/cr.2010.164; published online 30 November 2010

\section{Dear Editor,}

Ectopic expression of four transcription factors, including Oct4, Sox2, Klf4 and c-Myc, in differentiated fibroblast cells could reset the cell fate of fibroblast cells to pluripotent state [1]. Subsequently, full pluripotency of these so-called induced pluripotent stem cells (iPSCs) has been demonstrated, as viable mice could be generated autonomously from iPSCs through tetraploid blastocyst complementation [2, 3]. Moreover, the generation of human and patient-specific iPSCs has raised the possibility of utilizing iPSCs clinically [4-6]. However, the utilization of c-Myc in iPSC induction greatly increased the incidence of tumorigenecity in the iPS-chimeric mice and also might hinder the clinical application of human iPSCs in the future [7]. Fortunately, c-Myc has been recently found dispensable for iPSC induction even though the induction efficiency is greatly reduced in the absence of c-Myc [7]. However, it remains unknown whether these three factor-induced iPSCs are fully pluripotent. In the present study, we have successfully demonstrated that three-factor iPSCs could also be fully pluripotent, as viable mice could be generated from three-factor iPSCs autonomously via tetraploid complementation and moreover, our data indicate that the pluripotency regulatory mechanism in three-factor iPSCs might be distinct from four-factor iPSCs.

As previous described, we used Tet-on-inducible lentiviruses to transduce cDNAs of the three transcription factors Oct4, Sox 2 and Klf4 into mouse embryonic fibroblast (MEF) cells to generate three-factor iPSCs $[8,9]$. The MEF cells were collected from the 13.5 d.p.c. embryos, which were generated by mating the OG2 (Oct4GFP transgenic) mice with ROSA26-M2rtTA transgenic mice. After lentiviral infection, the MEF cells were cultured for 3 weeks in ES culture medium supplemented with doxycycline, subsequently, GFP-positive colonies appeared and then the doxycycline was withdrawn from the culture medium. After 3 days, ES-like colonies were picked and propagated individually (Figure 1A). In total, eight three-factor iPS cell lines were successfully established and further characterized. All cell lines exhibited morphology resembling normal ES cell lines with Oct4 expression demonstrated by GFP reporter (Figure 1A) and with normal karyotype (Supplementary information, Figure S1). RT-PCR and qPCR analysis further demonstrated that the expression of pluripotency marker genes in three-factor iPS cell lines appeared comparable to normal ES cell lines, but showed dramatic differences from the MEF cells (Figure 1B and Supplementary information, Figure S2). Immunofluorescent staining results further showed that these three-factor iPSCs positively expressed pluripotency transcription factors OCT4, NANOG and the ES cell surface marker SSEA1 (Figure 1C). Bisulphite genomic sequencing was further performed to analyze the methylation status of the Oct4 and Nanog promoters in the three-factor iPSCs, and the results confirmed that DNA demethylation occurred during the reprogramming process (Figure 1D). Genomic PCR for the exogenous factors further confirmed the insertion of the three transgenes in the genome without the transgenic c-Myc (Supplementary information, Figure S3). Taken together, the iPS cell lines derived using three transcription factors were similar to ES cells at the molecular level.

To further evaluate the functional pluripotency of the three-factor iPS cell lines, in vivo differentiation into teratomas and tetraploid blastocyst complementation assay were performed. The three-factor iPSCs were subcutaneously transplanted into immune-deficient SCID mice, and the formation of teratomas containing derivatives of all three germ layers could be observed 4 weeks after transplantation (Figure 1E). Tetraploid complementation assay was further performed to examine whether the three-factor iPSCs could capture the full pluripotency as observed in four-factor iPSCs. Generation of tetraploid blastocysts was essentially described in our recent study. Microinjection of 10 to 15 three-factor iPSCs into each tetraploid blastocyst was performed using a Piezo-drill micromanipulator. Subsequently, the tetraploid complemented embryos were transplanted into 
A

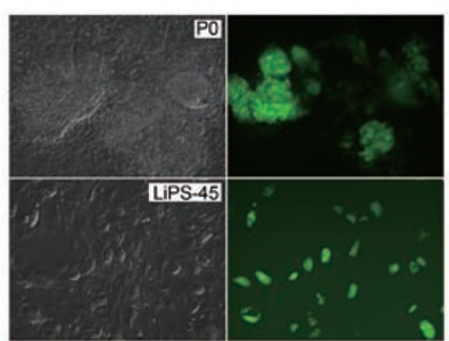

D

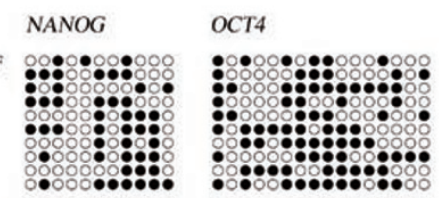

LiPS-45

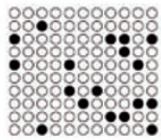

LiPS-57

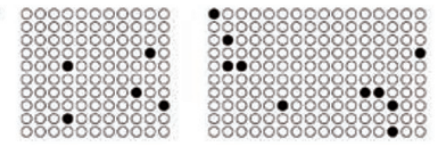

RI

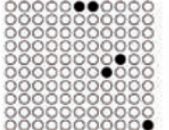

9800000
B

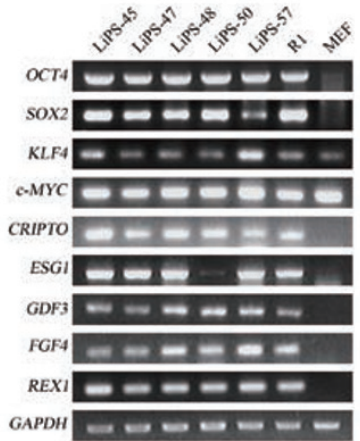

E

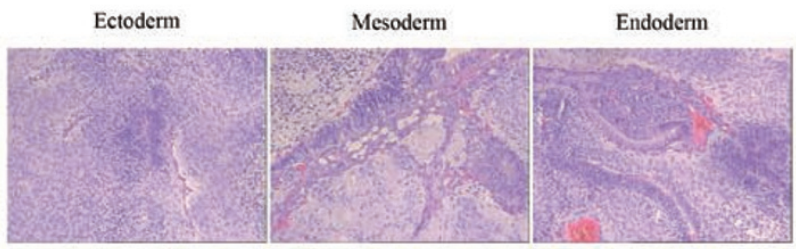

$\mathbf{F}$

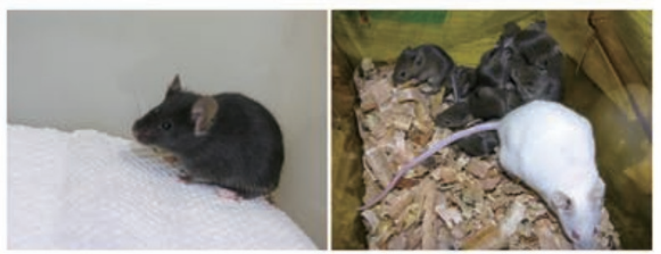

C

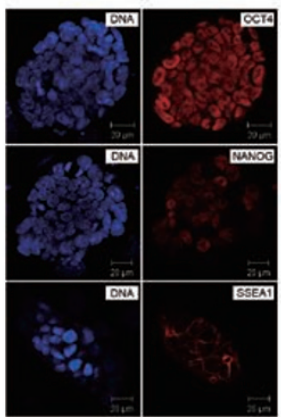

D2Mit102 $=\left\{\begin{array}{l}\text { pans } \\ \text { c5nit. }\end{array}\right.$
G
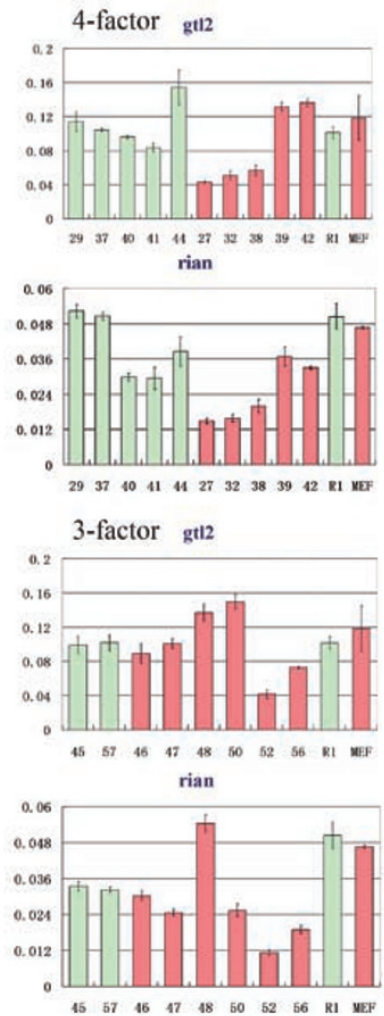

H

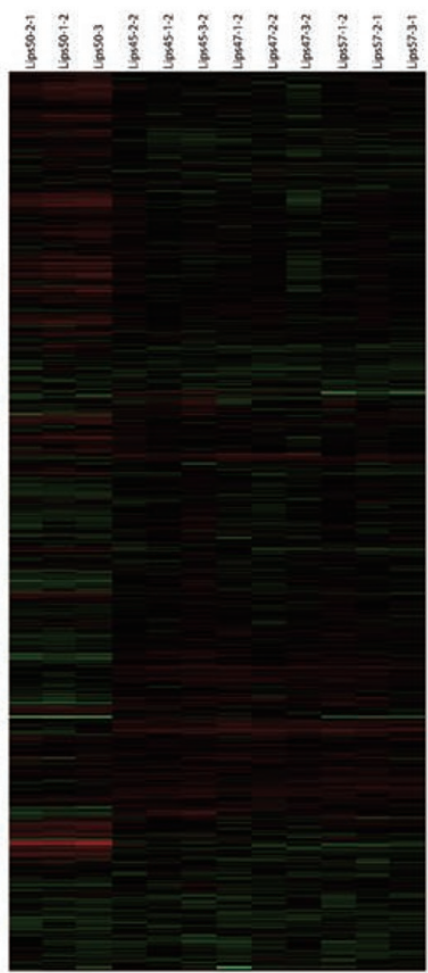

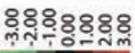

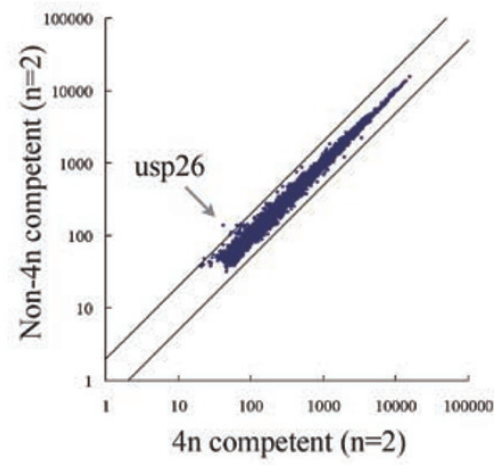

J

usp26

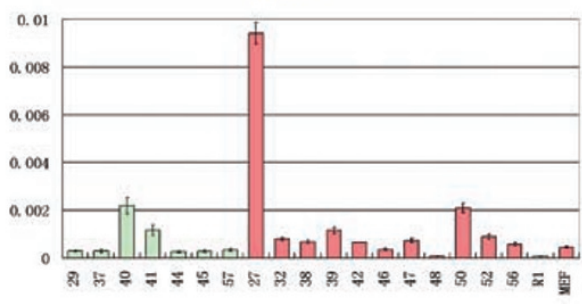


either oviduct or uterus of a pseudo-pregnant female ICR mouse. Development of the tetraploid complemented embryos into live embryos was observed by visualizing the Oct4-GFP expression in the genital ridges of an E13.5 embryo (Supplementary information, Figure S4). Moreover, viable full-term mice were successfully obtained from two three-factor iPS cell lines, namely LiPS-45 and LiPS-57, through this most rigorous test (Supplementary information, Figure S5). Furthermore, the mouse (named "little tiger", which was born on Chinese New Year) produced from LiPS-45 grows into adulthood and its germline transmission ability was further verified by crossing with ICR female mouse (Figure 1F). The genotyping of transgene rtta and simple sequence length polymorphism (SSLP) analysis further confirmed the identity of the three-factor iPS mouse. It showed the same background as the donor iPSCs, which contain $\mathrm{C} 57 \mathrm{BL} / 6 \mathrm{~J}$ genetic background with rtta insertion rather than the recipient embryos, which were B6D2F2 with C57BL/6J and DBA/2 genetic background without rtta insertion (Figure 1F). Therefore, for the first time, we demonstrated that iPSCs reprogrammed by three transcription factors in the absence of c-Myc could also capture full pluripotency as viable mice could be produced through tetraploid complementation. Omission of the oncogene c-Myc in reprogramming did not impair the property of the derived iPSCs.

It has been recently proposed that the imprinted Dlk1 cluster genes play crucial roles in determining the pluripotency of iPSCs reprogrammed by four transcription factors. Recent findings indicated that expression level of Dlk1 cluster genes in the iPS cell lines with full pluripotency was similar to ES cells, whereas the expression level of the cluster genes in the other iPS cell lines with inferior pluripotency was always significantly lower [10]. We next sought to examine these genes' expression in our four-factor iPS cell lines that we previously established as well as the threefactor iPS cell lines established in the present study. Our results showed that the expression level of these genes in the four-factor iPSCs was consistent with their ability to fulfill the tetraploid embryo complementation assay in general, although several exceptions were observed. Surprisingly, the expression level of these genes in most three-factor iPS cell lines showed no direct correlation with their pluripotency. Expression of these genes with similar level to ES cells was observed in most threefactor iPS cell lines (Figure 1G). Therefore, we propose that the molecular pluripotency regulatory mechanism might differ between four-factor- and three-factorinduced reprogramming.

To further explore the gene expression differences between the three-factor iPS cell lines with and without full pluripotency, we analyzed their global gene expression profile using microarray analysis, and the array data can be found in GEO database as GSE24046. Two three-factor iPS cell lines with full pluripotency and two lines with limited pluripotency were used for microarray analysis. Similar to the four-factor iPS cell lines previously reported, no obvious differences in global gene expression were distinguished between the two groups of cells (Figure 1H), and moreover we did not detect any difference in the expression of Dlk1 cluster genes between these two groups of cells. Interestingly, the deubiquitination enzyme, Usp26, was the only differentially expressed gene distinguished between these two groups of iPSCs (Figure 1I). Expression level of Usp26 in the three-factor iPS cell lines with full pluripotency was generally lower as compared with the lines without full pluripotency (Figure 1J), but some exceptions were also observed. Although the function of Usp26 in iPSCs pluripotency regulation remains elusive, we presume that the molecular regulatory mechanism in determining the pluripotency of three-factor iPSCs

Figure 1 Generation and characterization of the three-factor iPSCs. (A) Three-factor-derived iPSC colonies (upper) and stable cell line LiPS-45 (lower) both of which were GFP-positive indicating the OCT4 expression. (B) RT-PCR revealed that iPSCs expressed ES cell marker genes. (C) Immunofluorescent staining results demonstrated that iPSCs were positive for OCT4, NANOG and SSEA-1. (D) Bisulfite genomic sequencing of the promoter regions of Oct4 and Nanog in LiPS-45, LiPS-57 and MEF. Open and close circles indicate unmethylated and methylated CpGs, respectively. (E) Teratomas formed 4 weeks after injection of iPSCs into SCID mice. Tissues of the three germ layers were detected by hematoxylin and eosin staining. (F) The mouse derived totally from LiPS-45 developed into adulthood, and showed germline transmission capability. Genomic PCR and SSLP were performed to analyze the live adult mouse derived from iPS-tetraploid complementation. Transgene rtTA and D2Mit102 were analyzed. (G) Real time-PCR analysis of Gt12 and Rian expression in the four-factor (upper) and three-factor (lower) iPS cell lines. The green and red columns represented the lines with and without full pluripotency, respectively (the iPS cell lines with different pluripotency were shown in Supplementary information, Table S1). (H) Cluster comparing among $4 n$ competent and $4 n$ non-competent iPS cell lines. (I) Scatter plots comparing global gene expression patterns between $4 n$ competent and $4 n$ non-competent three-factor iPS cell lines, as determined by DNA microarray. Usp26 showed lower expression in $4 n$ competent three-factor iPS cell lines. (J) Real time-PCR analysis of the Usp26 expression level in the three-factor-derived iPS cell lines. 
might be different from four-factor iPSCs. More in-depth investigations will be needed to further elucidate the mechanism responsible for establishing the pluripotency regulatory network in three-factor iPSCs.

In conclusion, our present study demonstrated that iPSCs reprogrammed by three transcription factors in the absence of c-Myc could fully capture plurpotency and generate iPS mice through tetraploid complementation. Moreover, the pluripotency regulatory mechanism determining the pluripotency of three-factor iPSCs might differ from four-factor iPSCs.

\section{Acknowledgments}

We are grateful to our colleagues in our laboratory for their assistance with experiments and in the preparation of this manuscript. This project was supported by the Ministry of Science and Technology (grants 2008AA022311, 2010CB944900 and 2011CB964800).

Lan Kang ${ }^{1}$, Tong $\mathrm{Wu}^{1}$, Yu Tao ${ }^{1}$, Ye Yuan ${ }^{1}$, Jing $\mathrm{He}^{1}$, Yu Zhang ${ }^{1}$, Tong Luo ${ }^{1}$, Zhaohui Kou ${ }^{1}$, Shaorong Gao ${ }^{1}$

${ }^{I}$ National Institute of Biological Sciences, Beijing 102206, China

Correspondence: Shaorong Gao

Tel: +86-10-8072-8967; Fax: +86-10-8072-7535

E-mail: gaoshaorong@nibs.ac.cn

\section{References}

1 Takahashi K, Yamanaka S. Induction of pluripotent stem cells from mouse embryonic and adult fibroblast cultures by defined factors. Cell 2006; 126:663-676.

2 Kang L, Wang J, Zhang Y, Kou Z, Gao S. iPS cells can support full-term development of tetraploid blastocyst-complemented embryos. Cell Stem Cell 2009; 5:135-138.

3 Zhao XY, Li W, Lv Z, et al. iPS cells produce viable mice through tetraploid complementation. Nature 2009; 461:86-90.

4 Takahashi K, Tanabe K, Ohnuki M, et al. Induction of pluripotent stem cells from adult human fibroblasts by defined factors. Cell 2007; 131:861-872.

$5 \mathrm{Yu}$ J, Hu K, Smuga-Otto K, et al. Human induced pluripotent stem cells free of vector and transgene sequences. Science 2009; 324:797-801.

6 Dimos JT, Rodolfa KT, Niakan KK, et al. Induced pluripotent stem cells generated from patients with ALS can be differentiated into motor neurons. Science 2008; 321:1218-1221.

7 Nakagawa M, Koyanagi M, Tanabe K, et al. Generation of induced pluripotent stem cells without Myc from mouse and human fibroblasts. Nat Biotechnol 2008; 26:101-106.

8 Brambrink T, Foreman R, Welstead GG, et al. Sequential expression of pluripotency markers during direct reprogramming of mouse somatic cells. Cell Stem Cell 2008; 2:151-159.

9 Stadtfeld M, Maherali N, Breault DT, Hochedlinger K. Defining molecular cornerstones during fibroblast to iPS cell reprogramming in mouse. Cell Stem Cell 2008; 2:230-240.

10 Stadtfeld M, Apostolou E, Akutsu H, et al. Aberrant silencing of imprinted genes on chromosome $12 \mathrm{qF} 1$ in mouse induced pluripotent stem cells. Nature 2010; 465:175-181.

(Supplementary information is linked to the online version of the paper on the Cell Research website.) 